\title{
Cystic Pancreatic Lesions: A Review of Diagnosis and Management
}

\author{
Ott Le ${ }^{1} \quad$ Juan J. Ibarra Rovira ${ }^{1}$
}

1Department of Abdominal Imaging, The University of Texas MD

Anderson Cancer Center, Houston, Texas, United States

\begin{abstract}
Address for correspondence Ott Le, MD, Department of Abdominal Imaging, The University of Texas MD Anderson Cancer Center, 1515 Holcombe Boulevard, Unit 1473, Houston, TX 77030-4009, United States (e-mail: Ott.le@mdanderson.org).
\end{abstract}

\begin{abstract}
Keywords

- pancreatic cysts

- pseudocysts

- intraductal papillary mucinous neoplasm

- mucinous cystic neoplasm

- serous cystadenoma

Pancreatic cysts are commonly seen with increasing use of cross-sectional imaging. They range from a benign inflammatory process that can produce pseudocysts to malignant lesions such as mucinous cystadenocarcinoma. Other common pancreatic cysts include intraductal papillary mucinous neoplasms and serous cystadenomas. Optimized imaging protocol dedicated to imaging the pancreas is required, such as multiphasic computed tomography, magnetic resonance imaging/magnetic resonance cholangiopancreatography, or endoscopic ultrasound, to fully detect and characterize the lesions. A confident diagnosis can be made on imaging when features such as calcifications, pancreatic duct diameter, main duct communication, and mural nodules are assessed. Additionally, pathologic evaluation from fluid/tissue sampling aid in diagnosis. Optimal management of pancreatic cysts is achieved based on the imaging features, conveying key findings in the radiology report, pathologic evaluation, and clinical factors.
\end{abstract}

\section{Introduction}

It is common to see cystic pancreatic lesions, mostly due to the widespread use of cross-sectional imaging such as computed tomography (CT) or magnetic resonance imaging (MRI) and also the organ's location in the retroperitoneum, surrounded by fat. There are a variety of cystic pancreatic lesions, the most commonly reported as being the pseudocyst resulting from an inflammatory process. However, other cystic lesions such as side branch intraductal papillary mucinous neoplasm (IPMN) are increasingly reported with the greater use of thinner cross-sectional imaging. Cystic pancreatic lesions include a broad differential such as serous cystadenoma, mucinous cystadenoma/cystadenocarcinoma, and intraductal papillary mucinous tumor.

Approximately $2.5 \%$ of the general population has a cystic lesion in the pancreas between ages 40 and 84 years. The older the person is, the higher the percentage of occurrence. Approximately 2.2\% of all CT images of the abdomen and $20 \%$ of all MRIs of the abdomen will contain incidental pancreatic cystic lesions. The management of such findings is more complex than previous thought.
The American College of Radiology (ACR) is attempting to simplify concepts as well as help in the management of pancreatic cystic lesions according to size, patient age, and risk stratification by imaging. ${ }^{1}$

\section{Imaging Evaluation}

Pancreatic cysts are often incidental findings on CT performed for various other reasons. Further evaluation with an appropriate CT or MRI protocol are often necessary for optimal characterization; which typically consists of a dedicated pancreatic protocol multiphasic CT, MRI/magnetic resonance cholangiopancreatography (MRCP), or endoscopic ultrasound (EUS). A dedicated CT study with pancreatic protocol consists of a typically precontrast component and effective late arterial and porta venous phases obtained approximately 40 to 50 seconds and 70 to 80 seconds, respectively, after contrast injection. ${ }^{2}$ This is the typical pancreatic phase (late arterial phase), unlike the early arterial phase which obtain images around 30 seconds after contrast injection. ${ }^{3}$ The late arterial phase provides optimal differentiation between normal pancreatic parenchymal and hypodense lesions
DOI https://doi.org/ 10.1055/s-0040-1708107 ISSN 2581-9933.
License terms

(이 (1) $\Theta \circledast$ 
and provides good opacification of the arterial and venous vessels. ${ }^{2}$ MRI has been shown to be better than CT in differentiating IPMN from other cystic pancreatic lesions (96.8 vs. $80.6 \%$ sensitivity and 90.8 vs. $86.4 \%$ specificity). ${ }^{4}$ Furthermore, consideration should be made to obtain MRI of the abdomen for the younger population, since the ACR algorithm requires follow-up exams for up to 10 years with different intervals depending on age and lesion size. T2-weighted imaging using breath hold fast spin echo (FSE) or free breathing single shot fast spin echo (SSFSE) techniques are important in axial and coronal planes for better assessment. The use of contrast is also recommended

Additionally, optimal imaging often consists of thin-section imaging and appropriate postprocessing techniques, which is especially needed in determining whether there is cystic communication with the pancreatic duct. Multiplanar reformatting (MPR) and curved MPR (cMPR), alone or in combination with oblique axial images, are superior to axial images when evaluating the pancreatic duct as shown by some studies..$^{5-7}$ Also, another postprocessing technique is minimal intensity projection (MinIP), which may enhance the detection of cystic lesions, their internal characteristics and communication with the pancreatic ducts if any is present. $^{7}$

Conveying key features identified on imaging via the radiology report is crucial for patient management. Therefore, the ACR encourages reporting worrisome features and high-risk stigmata, as well as any change such as interval growth, as listed in $\mathbf{r}$ Tables $\mathbf{1}$ and $\mathbf{2}$. Growth of lesions remain the most widely use parameter for surveillance ( - Table 3 ).

\section{Pathologic Evaluation}

Since all cystic lesions in the pancreas are presumed mucinous unless definite history of pancreatitis or previous biopsy was performed, fluid/tissue sampling is considered when CT or MRI features are worrisome or nonspecific.

The goal is to obtain a 2-cc sample submitted for a panel of basic laboratories and staining (-Table 4). The lesion

Table 1 Worrisome features of cystic pancreatic lesions

\begin{tabular}{|l|}
\hline Lesion $>3 \mathrm{~cm}$ \\
\hline Thickened wall \\
\hline Nonenhancing mural nodule \\
\hline Main pancreatic duct $>7 \mathrm{~mm}$ \\
\hline
\end{tabular}

Table 2 High-risk stigmata of cystic pancreatic lesions

\begin{tabular}{|l|}
\hline Enhancing mural nodule \\
\hline Main pancreatic duct $>10 \mathrm{~mm}$ \\
\hline Clinical obstructive jaundice \\
\hline
\end{tabular}

Table 3 Growth of lesions surveillance

\begin{tabular}{|l|}
\hline Lesion $<0.5 \mathrm{~cm}$ must have $100 \%$ increase in long axis diameter \\
\hline Lesion $0.5-1.5 \mathrm{~cm}$ must have $50 \%$ increase in long diameter \\
\hline Lesion $>1.5 \mathrm{~cm}$ needs $20 \%$ increase in long diameter \\
\hline
\end{tabular}

Table 4 Sample evaluation, basic laboratories, and staining

\begin{tabular}{|l|}
\hline Alcian blue stain for mucin \\
\hline carcinoembryonic antigen value \\
\hline Amylase value \\
\hline Cytology \\
\hline
\end{tabular}

must be at least 1.7 to $2 \mathrm{~cm}$ in maximum dimension and the yield dependent on the endoscopic expertise of the gastroenterologist.

Combined with optimal imaging, the sensitivity and specificity of diagnosis ranges between 90 to $100 \%$ and 92 to $98 \%$, respectively.

\section{Pseudocysts}

The most common cystic pancreatic lesion has reportedly been a pseudocyst (up to $80 \%$ ), ${ }^{8}$ therefore the presence of previous inflammatory history is highly relevant.

Pseudocysts develop after approximately 4 weeks from a single- or multiple-acute episode of edematous pancreatitis. They are homogeneous in density and well circumscribed and need to be differentiated from wall off necrosis (WON), which can develop after necrotizing pancreatitis ( - Fig. 1). The latter is usually more inhomogeneous and higher in density due to necrotic tissue or blood products. ${ }^{9}$

Chronic pancreatitis can display imaging features overlapping with those of main duct IPMN, as both can present with main pancreatic ductal dilatation and parenchymal atrophy. Nevertheless, imaging features favoring chronic pancreatitis include ductal and parenchymal stones and lack of enhancing mural nodules or a bulging papilla. ${ }^{8}$

Again, the increasing use of thinner cross-sectional imaging is revealing cystic pancreatic lesions other than pseudocysts, such as IPMN, mucinous cystic neoplasm (MCN), or serous cystadenoma.

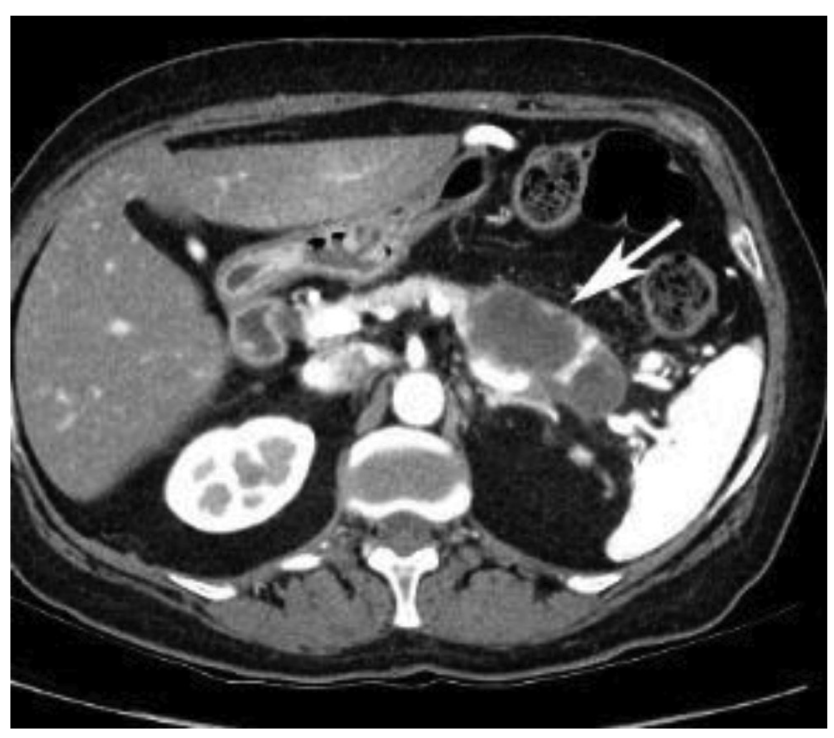

Fig. 1 CT demonstrates pseudocyst (white arrow) in patient with history of chronic pancreatitis, pathology confirmed. CT, computed tomography. 


\section{Intraductal Pancreatic Mucinous Neoplasm}

The most common resected cystic lesions of the pancreas are IPMNs, accounting for approximately $50 \%$ of cases. They are more prevalent in men and typically occur in the sixth or seventh decade. These tumors can originate from the main pancreatic duct or its side branch; being classified as main duct, side branch, or combined tumors. The side branch is the most common variant. They can present histologically from benign dysplasia, borderline malignancy, to infiltrative carcinomas. ${ }^{8}$ Features seen on CT described as predictive of increased malignant risks include main pancreatic ductal dilatation greater than $10 \mathrm{~mm}$, diffuse or multifocal involvement, calcified intraluminal content, bulging papilla, and solid contrast enhancing papillary proliferations. ${ }^{8}$ Main duct forms of IMPN are at a higher incidence of malignant transformation than side branch varieties, 38 to $68 \%$ vs. 12 to $47 \%$. However, side branch IPMN measuring larger than $3 \mathrm{~cm}$ or growth of greater than $2 \mathrm{~mm} / \mathrm{year}$ is associated with increased malignancy risks as shown by some studies. ${ }^{4}$ Those lesions without the characteristics of increased malignant risks show a trend toward observation as described in different guidelines, such as from the ACR or publications by Tanaka et al. ${ }^{1,10}$ As stated previously, chronic pancreatitis can have imaging features that overlap with those of main duct IPMN, as both can present with main pancreatic ductal dilatation and parenchymal atrophy. Nevertheless, imaging features again favoring chronic pancreatitis include ductal stones and lack of enhancing ductal wall nodules or a bulging papilla. ${ }^{8}$

It is important to identify the communication of the cystic neoplasm to the main pancreatic duct to secure the diagnosis of a side branch or side duct IPMN, $\boldsymbol{-}$ Fig. $\mathbf{2}$ and $\boldsymbol{-}$ Fig. $\mathbf{3}$. If no communication is visualized in a well-performed study, MCN is more likely and accounts for approximately $25 \%$ of all cystic resected lesions.

\section{Mucinous Cystic Neoplasm}

MCN of the pancreas are typically referred as "mother cysts" as they occur in women in the fourth to fifth decade. Unlike serous cystadenoma, its histology ranges from a benign adenoma and borderline tumor to noninvasive and invasive carcinoma. They present as either a unilocular or multilocular macrocystic lesion as seen on CT. Imaging characteristics concerning for malignant potential include the presence of a thick enhancing septate, solid mural papillary projections, or partially solid component. The cyst

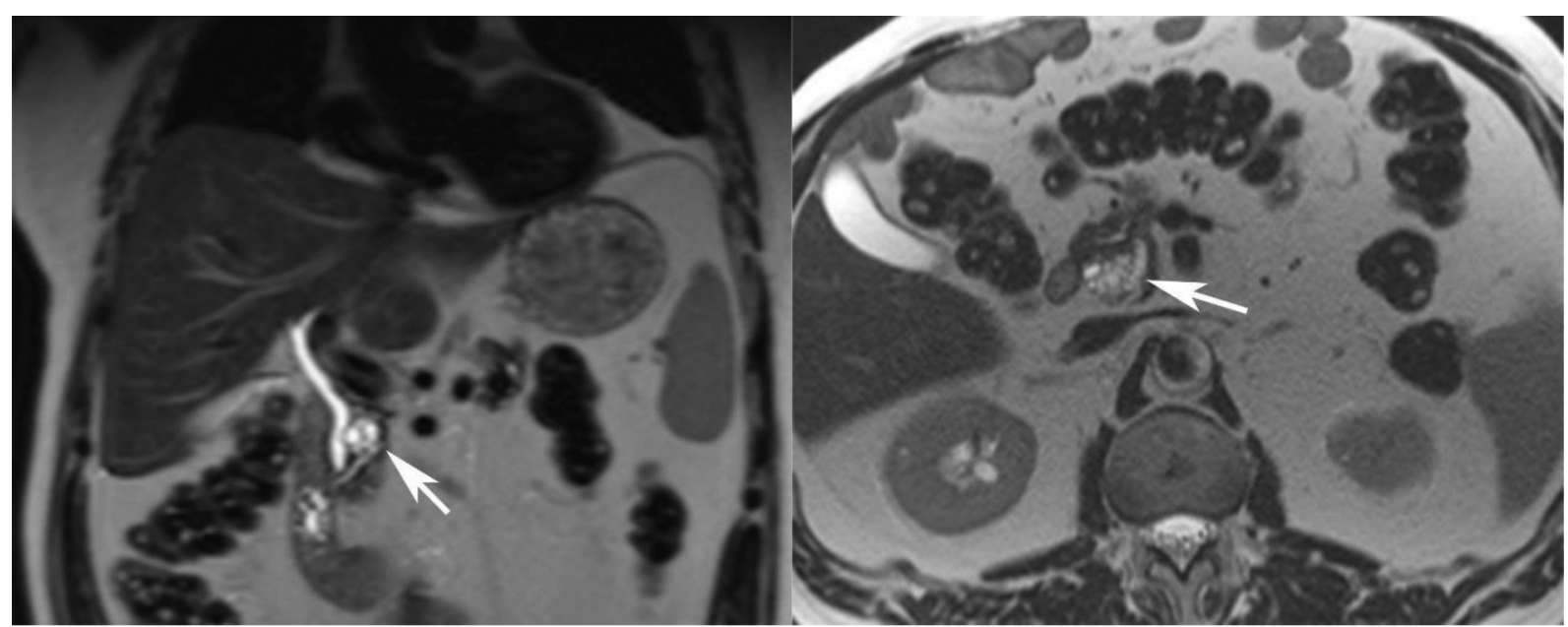

Fig. 2 Multicystic lesion with communication to MPD (white arrow) compatible with side branch IPMN on MRI, confirmed on cytology. IPMN, intraductal pancreatic mucinous neoplasms; MPD, main pancreatic duct; MRI, magnetic resonance imaging.

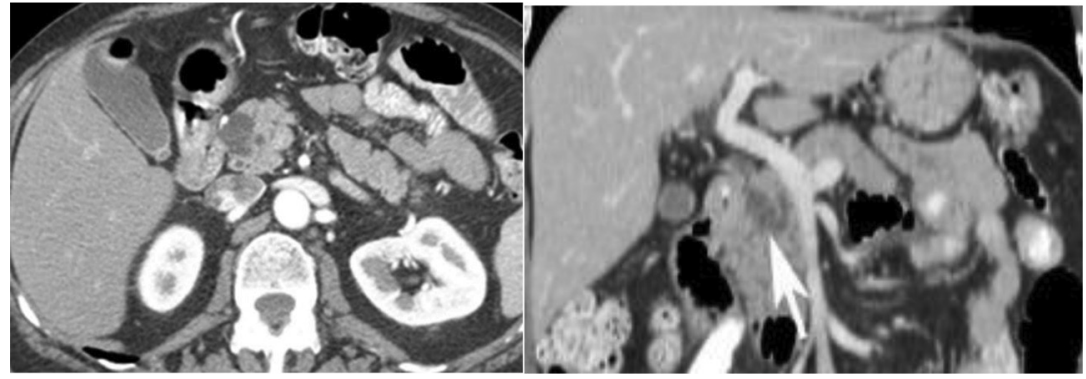

Fig. 3 Typical appearance of a side branch IPMN on CT with MPD communication (white arrow), confirmed on cytology. CT, computed tomography; IPMN, intraductal papillary mucinous neoplasm; MPD, main pancreatic duct. 


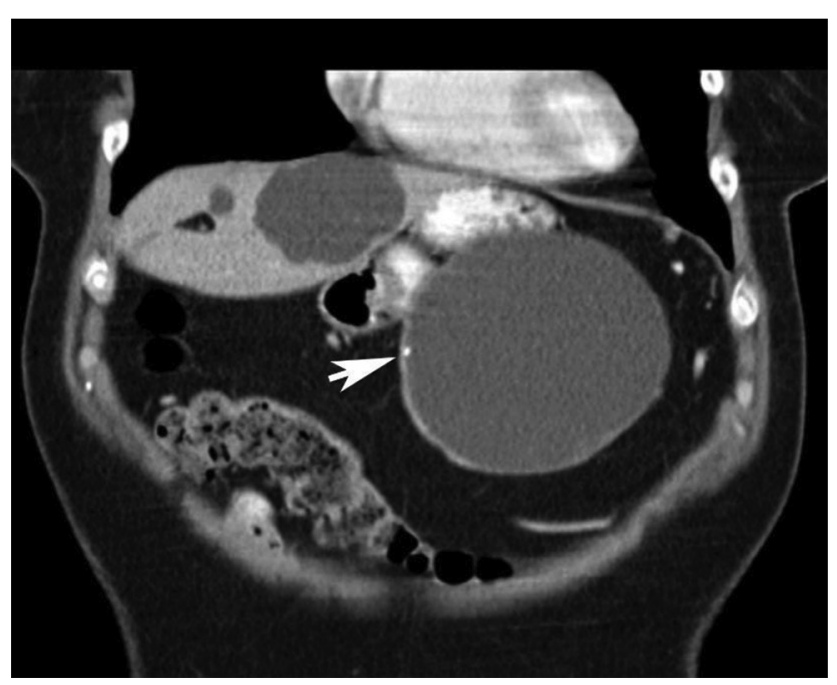

Fig. 4 CT demonstrates large cystic pancreatic mass with peripheral calcification (white arrow), confirmed MCN. MCN, mucinous cystic neoplasm.

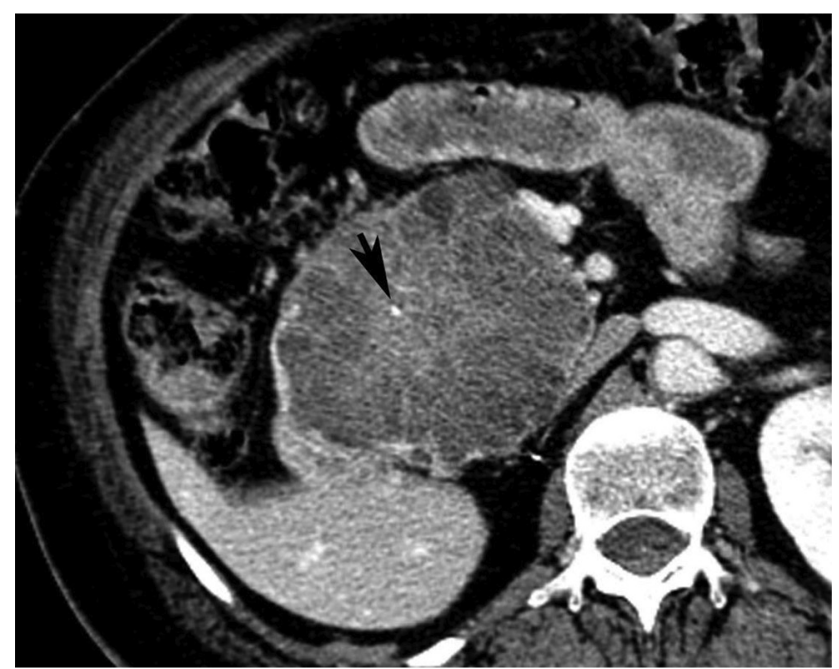

Fig. 5 CT demonstrates large cystic pancreatic mass with central calcification (black arrow), confirmed serous cystadenoma.

may have wall calcifications, which distinguishes these lesions from the centrally calcified serous cystadenomas ( - Fig. 4). As stated before, a lack of communication with the main pancreatic duct or side branch helps differentiate these cysts from an IPMN.

\section{Serous Cystadenoma}

Serous cystadenoma of the pancreas is generally benign and has been referred as "grandmother cysts" as they commonly affect women in the sixth to seventh decade. On CT imaging, they are comprised of small multiple typically greater than six cysts, each less than $2 \mathrm{~cm}$ in diameter and do not communicate with the pancreatic duct. When larger, serous cystadenomas are seen as sponge-like mass (microcystic) with a central punctate or globular calcification ( - Fig. 5). ${ }^{8}$ They can also present as a unilocular mass rarely, which can be indistinguishable from a mucinous cystadenoma or cystadenocarcinoma. Further evaluation with EUS with biopsy may be of benefit.

\section{Other Features}

Additionally, there are other imaging features that can aid in charactering cystic pancreatic lesions. Measuring cyst density (Hounsfield's unit [HU]), is an example; as reported by Chalian et al, cysts with attenuation greater than $14.5 \mathrm{HU}$ are more associated with pseudocysts than unilocular mucin-containing cysts (accuracy of 73.5\%). ${ }^{11}$ Benign pancreatic cysts also more commonly exhibit a lobulated shape, thin wall and smooth internal surface; in contrast to premalignant and malignant lesions demonstrating often a round, oval, or complex shape with thick wall, and irregular internal surface. ${ }^{12}$ As previously described, EUS-guided cyst fluid aspiration can further help to characterize pancreatic cysts; mucinous tumors will often yield elevated levels of carcinoembryonic antigen (CEA), or carbohydrate antigen 19 to 9 (CA19-9) and increased fluid viscosity. ${ }^{13}$

\section{Management}

Management of cystic pancreatic lesions depends on optimal imaging and characterization (-Fig. 6). A cystic lesion characteristic of serous cystadenoma is not surgically resected unless $4 \mathrm{~cm}$ or more in size and symptomatic. ${ }^{14}$ On the contrary, imaging features suggestive of MCN are resected in suitable candidates, regardless of size secondary to their malignant potential. ${ }^{4}$ IPMN tumors less than $3 \mathrm{~cm}$ in size and simple in appearance can be followed according to the 2012 international consensus guidelines from the International Association of Pancreatology in Fukuoka, Japan; a 2- to 3-year follow-up for lesions less than $10 \mathrm{~mm}$, yearly for lesions 10 to $20 \mathrm{~mm}$, and 3 to 6 months for lesions 20 to $30 \mathrm{~mm}^{.10}$

\section{Conclusion}

Pancreatic cysts are now commonly seen with increasing use of cross-sectional imaging. They range from a benign inflammatory process that can produce pseudocysts to malignant lesions such as mucinous cystadenocarcinoma. Optimized imaging protocol dedicated to imaging the pancreas is required, such as multiphasic CT, MRI/MRCP, or EUS to fully detect and characterize the lesions. A confident diagnosis can be made on imaging when features such as calcifications, pancreatic duct diameter, main duct communication, and mural nodules are assessed. Additionally, pathologic evaluation from fluid/tissue sampling aid in diagnosis. Optimal management of pancreatic cysts is achieved based on the imaging features, pathologic evaluation, and clinical factors. 


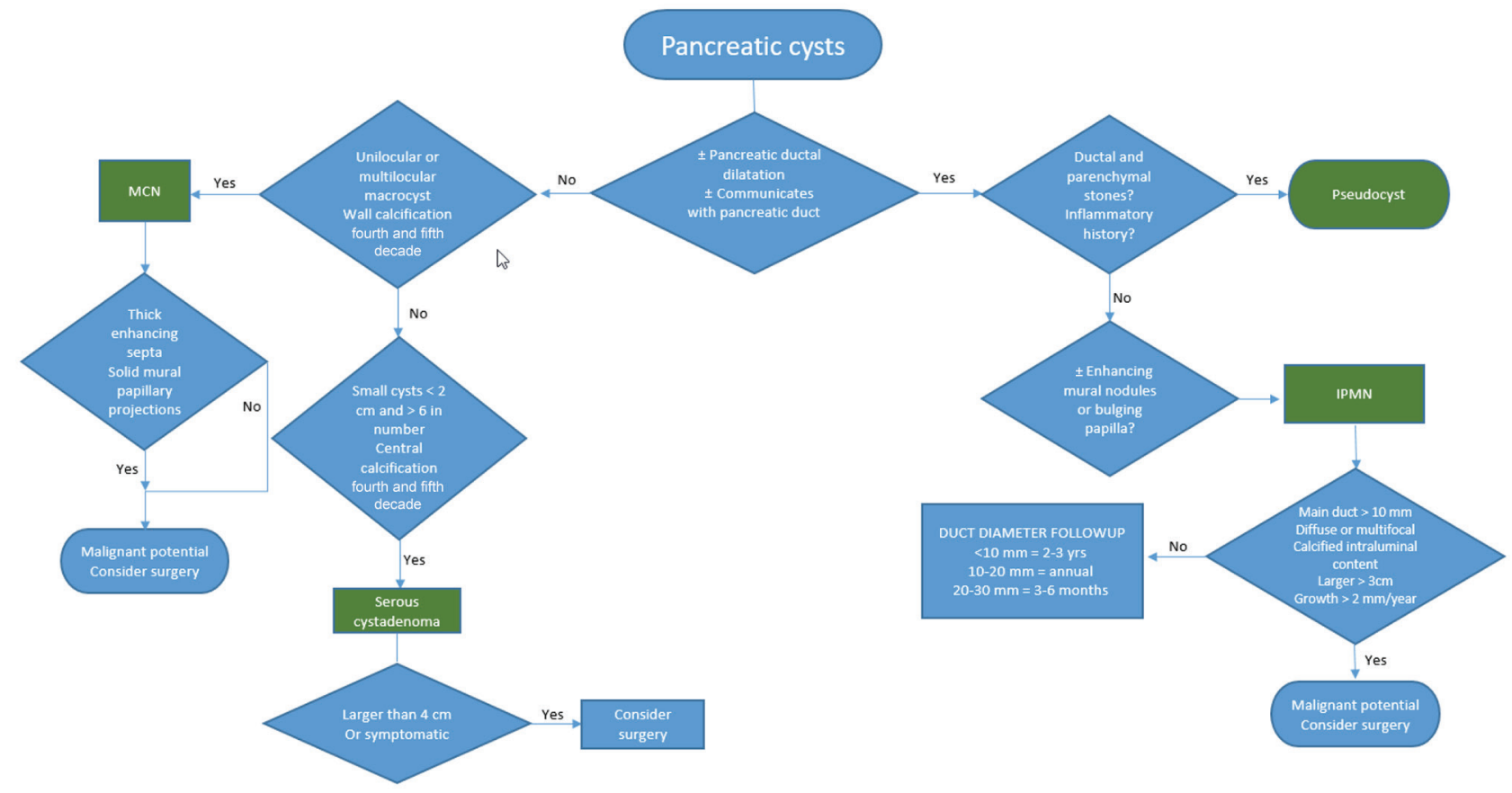

Fig. 6 Flow chart of pancreatic cysts for diagnosis and management. IPMN, intraductal pancreatic mucinous neoplasms; MCN, mucinous cystic neoplasm.

\section{Conflict of Interest}

None declared.

\section{Acknowledgments}

The authors wish to acknowledge Raghu Vikram, MD, for his support of this work.

\section{References}

1 Megibow AJ, Baker ME, Morgan DE, et al. Management of incidental pancreatic cysts: A white paper of the ACR incidental findings committee. J Am Coll Radiol 2017;14(7):911-923

2 Quencer K, et al. Imaging of the pancreas: part 1. Appl Radiol 2013;42(9):14-20

3 Callery MP, Chang KJ, Fishman EK. Talamonti MS, William Traverso L, Linehan DC. Pretreatment assessment of resectable and borderline resectable pancreatic cancer: expert consensus statement. Ann Surg Oncol 2009;16(7):1727-1733

4 Zaheer A, Pokharel SS, Wolfgang C, Fishman EK, Horton KM. Incidentally detected cystic lesions of the pancreas on CT: review of literature and management suggestions. Abdom Imaging 2013;38(2):331-341

5 Itoh S, Ikeda M, Ota T, Satake H, Takai K, Ishigaki T. Assessment of the pancreatic and intrapancreatic bile ducts using $0.5-\mathrm{mm}$ collimation and multiplanar reformatted images in multislice CT. Eur Radiol 2003;13(2):277-285

6 Fukushima H, Itoh S, Takada A, et al. Diagnostic value of curved multiplanar reformatted images in multislice CT for the detection of resectable pancreatic ductal adenocarcinoma. Eur Radiol 2006;16(8):1709-1718

7 Tamm EP, Balachandran A, Bhosale P, Szklaruk J. Update on 3D and multiplanar MDCT in the assessment of biliary and pancreatic pathology. Abdom Imaging 2009;34(1):64-74

8 Schima W, Kölblinger C, Ba-Ssalamah A, MDCT of pancreatic tumors, In: Reiser MF, Becker CR, Nikolaou K, Glazer G, eds. Multislice CT. 3rd ed. Berlin, Germany: Springer-Verlag Berlin Heidelberg; 2009 407-422

9 Foster BR, Jensen KK, Bakis G, Shaaban AM, Coakley FV. Revised Atlanta classification for acute pancreatitis: a pictorial essay-erratum. Radiographics 2019;39(3):912

10 Tanaka M, Fernández-del Castillo C, Adsay V, et al; International Association of Pancreatology. International consensus guidelines 2012 for the management of IPMN and MCN of the pancreas. Pancreatology 2012;12(3):183-197

11 Chalian H, Töre HG, Miller FH, Yaghmai V. CT attenuation of unilocular pancreatic cystic lesions to differentiate pseudocysts from mucin-containing cysts. JOP 2011;12(4):384-388

12 Pongpornsup S, Piyapittayanan S, Charoensak A. MDCT imaging findings for characterization pancreatic cystic lesion: differentiation between benign and malignant pattern. J Med Assoc Thai 2011;94(3):369-378

13 Edirimanne S, Connor SJ. Incidental pancreatic cystic lesions. World J Surg 2008;32(9):2028-2037

14 Tseng JF. Management of serous cystadenoma of the pancreas. J Gastrointest Surg 2008;12(3):408-410 